\title{
$\mathrm{C} / \mathrm{C}$ 编织复合材料双剪连接结构的挤压强度*
}

\author{
唐玉玲 ${ }^{1,2}$ 陈 浩 ${ }^{1}$ 周振功 ${ }^{2}{\text { 张 } \text { 琰 }^{1} \text { 郭 颖 }}^{2,3}$ \\ (1. 天津科技大学天津市轻工与食品工程机械装备集成设计与在线监控重点实验室 天津 300222; \\ 2. 哈尔滨工业大学复合材料与结构研究所 哈尔滨 150001 ;
}

3. 哈尔滨理工大学建筑工程学院 哈尔滨 150006)

\begin{abstract}
摘要: 对碳/碳 $(C / C)$ 编织复合材料双剪螺栓连接结构进行试验与数值研究。在拉伸载荷下对 $\mathrm{C} / \mathrm{C}$ 编织复合材料螺栓连接结构 的挤压接强度进行测试, 分析堆叠顺序、开孔直径和预紧力对失效模式和挤压强度的影响, 得到 $C / C$ 编织复合材料双剪螺栓 连接结构的失效模式, 给出结构的失效机制。采用 ABAQUS 建立连接结构的非线性接触模型, 利用垫片升温法实现螺栓预 紧力, 采用 VUMAT 子程序建立基于应变的 Hashin 破坏模型, 该模型可效预报 C/C 编织复合材料双剪螺栓连接结构的渐进 损伤、失效模式及挤压强度。利用该分析模型, 给出 $\mathrm{C} / \mathrm{C}$ 编织复合材料双剪螺栓连接结构的铺层方式和几何尺寸设计参考值, 并绘制了该类材料双剪螺栓连接结构的破坏包线图。
\end{abstract}

关键词: $\mathrm{C} / \mathrm{C}$ 编织复合材料; 螺栓连接; 强度; 破坏; 有限元分析

中图分类号: TB332

\section{Bearing Strength of Carbon / Carbon Braided Composites Double Shear Joint}

TANG Yuling $^{1,2}$ CHEN Hao $^{1}$ ZHOU Zhengong ${ }^{2}$ ZHANG Yan ${ }^{1}$ GUO Ying ${ }^{2,3}$

(1. Tianjin Key Laboratory of Integrated Design and On-line Monitoring for Light Industry\& Food

Machinery and Equipment, Tianjin University of Science \& Technology, Tianjin 300222;

2. Center for Composite Materials and Structures, Harbin Institute of Technology, Harbin 150001;

3. School of Architecture and Civil Engineering, Harbin University of Science and Technology, Harbin 150006)

\begin{abstract}
A comprehensive experimental and numerical evaluation of carbon/carbon (C/C) composites double-shear bolted joint is presented. The effects of the stacked sequence, bolt hole diameter and bolt-torque in joints are examined. Failure loads and failure modes of $\mathrm{C} / \mathrm{C}$ composites double-shear configurations subjected to tensile loading are obtained and compared. Failure mechanism is analyzed and discussed. A non-linear contact model is developed using ABAQUS. The bolt-torque is realized by heating gasket. A strain-based Hashin failure model is established using VUMAT subroutine. The model effectively predict the progressive damage, failure mode and bearing strength of the $\mathrm{C} / \mathrm{C}$ braided composite double-shear bolt joints. Based on the analysis model, the stacked sequence and geometric dimension design reference value of $\mathrm{C} / \mathrm{C}$ braided composite double-shear bolt joints are given, as well as the failure envelope is given.
\end{abstract}

Key words: carbon/carbon braided composites; bolted joint; strength; failure; finite element analysis

\section{0 前言}

碳/碳(C/C)复合材料在 $1400{ }^{\circ} \mathrm{C}$ 高温下能够较

* 国家自然科学基金(11272105)、天津市轻工与食品工程机械装备集成设 计与在线监控重点实验室开放基金(2017 LIMFE02, 2019 LIMFE04)和黑 龙江省教育厅科学技术(面上)(12541153)资助项目。20190810 收到初稿, 20200205 收到修改稿
好地保持结构强度 ${ }^{[1-4]}$, 是重要的热结构材料。NASA 的 Glenn 研究中心对热结构材料的连接和装配问题 进行了研究。分析了陶瓷基复合材料连接的研究背 景、工程实际需要、所需关键技术和基本原理, 对 连接件的设计(连接形式)、性能测试、数据分析以 及耐久性进行了讨论。由于热结构材料连接技术是 热结构设计的关键，因此国内外公开的资料与研究 成果非常有限。此外, $\mathrm{C} / \mathrm{C}$ 复合材料是一种脆性材 
料且各项异性, 材料自身拓扑结构复杂, 结构失效 模式多尺度、多重耦合, 这些都加大了其连接结构 力学性能分析的难度。

国内关于热结构材料连接性能的研究成果主 要针对 $\mathrm{C} / \mathrm{C}$ 和 $\mathrm{C} / \mathrm{SiC}$ 这两类复合材料的初步研究。 矫桂琼教授课题组对不同编织结构的 $\mathrm{C} / \mathrm{SiC}$ 复合 材料螺栓在室温环境下的拉伸、剪切、扭转的力 学性能进行了试验研究, 并对 $\mathrm{C} / \mathrm{SiC}$ 复合材料开 孔试件的力学性能进行了研究, 取得了一些规律性 的认识 ${ }^{[5-6]}$ 。周振功教授课题组在 $\mathrm{C} / \mathrm{C}$ 复合材料连 接件的基础理论与试验方面做了初步的研究工 作 ${ }^{[2,7]}$, 进行了 $\mathrm{C} / \mathrm{C}$ 复合材料单剪单孔和单剪双孔 连接拉伸性能的测试与失效机理分析, 揭示了 $\mathrm{C} / \mathrm{C}$ 复合材料单剪单孔和单剪双孔连接方式对连 接强度的影响规律。目前, 国内外针对复合材料 连接结构分析比较多的集中在对碳纤维树脂基复 合材料层板 (Carbon fiber reinforced polymer, CFRP)的连接性能研究 ${ }^{[8-14]}$, 其中包括连接几何尺 寸 $^{[9]}$ 、铺层顺序 ${ }^{[10]}$ 、间隙 ${ }^{[11]}$ 及预紧力 ${ }^{[12]}$ 等因素对 连接结构的连接性能及失效模式的影响。然而, $\mathrm{C} / \mathrm{C}$ 编织复合材料这类热结构材料与 CFRP 从材 料组分、制备工艺到力学性能有着显著的差异。 目前 $\mathrm{C} / \mathrm{C}$ 连接结构的试验数据非常有限, 更没有 建立系统的 $\mathrm{C} / \mathrm{C}$ 编织复合材料连接设计的理论方 法, 包括连接设计准则、连接设计的许用值确定、 连接强度分析方法、高温环境下的连接设计方 法等。

本文以 $\mathrm{C} / \mathrm{C}$ 编织复合材料双剪螺栓连接结构为 研究对象, 通过试验研究 $\mathrm{C} / \mathrm{C}$ 编织复合材料的挤压 强度, 给出其失效机制, 获得挤压强度与铺层、孔 径、侧向约束等影响因素之间的关系。建立有效 的数值模型, 减少连接性能分析中的物理试验, 为 $\mathrm{C} / \mathrm{C}$ 编织复合材料连接结构的设计提供试验及 理论依据。

\section{1 试验方法}

通常复合材料连接的失效模式有: 挤压破坏、 拉伸破坏、剪切破坏及混合破坏。设计者希望连接 结构发生挤压破坏或伴随挤压破坏的塑性失效模式。 因此挤压强度就是连接结构设计中非常重要的性能 指标。为了获得 $\mathrm{C} / \mathrm{C}$ 编织复合材料连接结构在面内 载荷下不同连接参数(铺层顺序、预紧力、几何尺寸 等)对其挤压强度的影响及结构失效机制, 对 $\mathrm{C} / \mathrm{C}$ 编 织复合材料进行挤压测试。
挤压测试试件均采用碳布层叠缝合工艺制备的 $\mathrm{C} / \mathrm{C}$ 编织复合材料平板, 平板纤维含量 $45 \%$ ～ $48 \%$ 。 碳布为 T300-3K 八枚缎, 缝合线为 $(\mathrm{T} 300-3 \mathrm{~K}+\mathrm{T} 300-$ $1 \mathrm{~K}) \times 2$, 缝合间距 $4 \mathrm{~mm}$, 增强体结构示意图如图 1a 所示(由试件制备单位提供)。为使连接发生挤压破 坏, 获得双剪挤压破坏机理, 根据 ASTM D5961 ${ }^{[15]}$ 标准, 参考文献[2]中单剪连接结构的几何设计值及 $\mathrm{C} / \mathrm{C}$ 编织复合材料平板板厚, 确定双剪连接结构孔 径 $D$ 为 $8 \mathrm{~mm}$ 和 $10 \mathrm{~mm}$ 两种, 端距 $E=30 \mathrm{~mm}$ 、板宽 $W=40 \mathrm{~mm}$ 。测试试件结构及夹具如图 $1 \mathrm{~b}$ 所示, 挤 压试件结构几何尺寸如表 1 所示, 每种工况测试 5 组试件。

表 $1 \mathrm{C} / \mathrm{C}$ 编织复合材料螺栓连接结构挤压试件

\begin{tabular}{llcc}
\hline 试件编号 & \multicolumn{1}{c}{ 堆叠顺序 } & $\begin{array}{c}\text { 扭转力矩 } T / \\
(\mathrm{N} \cdot \mathrm{m})\end{array}$ & $\begin{array}{c}\text { 开孔直径 } D / \\
\mathrm{mm}\end{array}$ \\
\hline B8-0-T0 & {$[0 / 90 / 45 / 0 / 90 /-45]_{4}$} & 0.5 & 8 \\
B10-0-T0 & {$[0 / 90 / 45 / 0 / 90 /-45]_{4}$} & 0.5 & 10 \\
B10-45-T0 & {$[45 /-45 / 90 / 45 /-45 / 0]_{4}$} & 0.5 & 10 \\
B10-0-T10 & {$[0 / 90 / 45 / 0 / 90 /-45]_{4}$} & 10 & 10 \\
B10-0-T20 & {$[0 / 90 / 45 / 0 / 90 /-45]_{4}$} & 20 & 10 \\
\hline
\end{tabular}

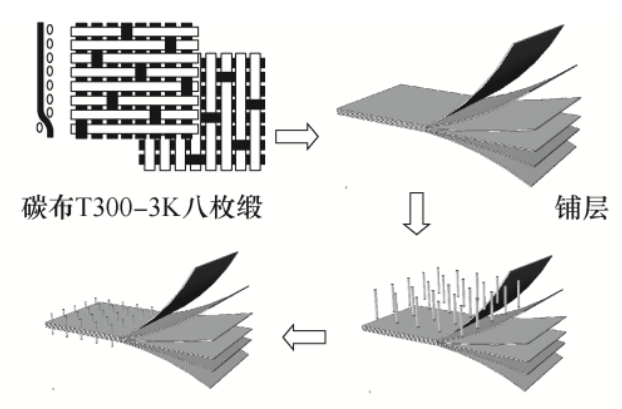

缝合后磨去表面多余缝合线缝合间距 $4 \times 4 \mathrm{~mm}$ (a) $\mathrm{C} / \mathrm{C}$ 编织复合材料制备示意图

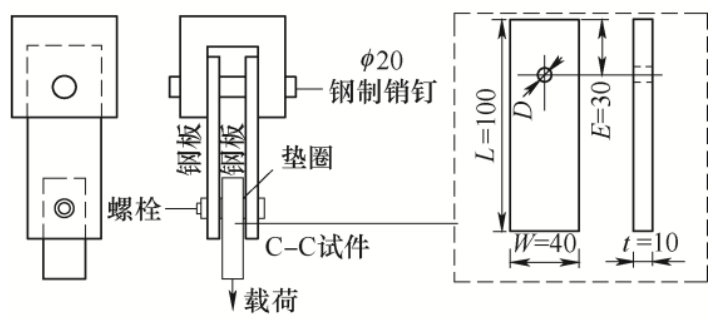

(b) 挤压测试夹具结构图及试件几何 $(\mathrm{mm})$

图 $1 \mathrm{C} / \mathrm{C}$ 编织复合材料螺栓连接结构挤压测 试材料、试件及夹具结构图

挤压测试试验装置如图 2 所示, 为了避免挤 压测试过程中试件被夹具破坏，在试件夹持端粘 贴 $1.5 \mathrm{~mm}$ 厚铝板作为加强片。采用数显扭矩 扳手 CNB20(量程 $20 \mathrm{~N} \cdot \mathrm{m}$, 精度等级 $\pm 1 \%$ ) 施加 预紧力。使用 INSTRON5569(量程 $50 \mathrm{kN}$ ) 试验 机进行测试, 试验在常温下进行, 加载速率为 
$0.5 \mathrm{~mm} / \mathrm{min}$, 测试过程中采用激光引申计测试结 构位移。

为了获得试件在加载过程中的完整失效过程, 在试件发生不同程度的破坏时终止加载。当挤压 试件的载荷分别达到第一与第二峰值时终止加载 (即图 3a 中的 $C 、 K$ 点)。无二次峰值的测试件在 载荷分别达到峰值与下降至峰值 $80 \%$ 的时刻终止 加载(即图 $3 \mathrm{~b}$ 中的 $C 、 K$ 点)。将试件利用水切割 沿破坏平面切开, 采用 OLYMPUS (SZX12)体式 显微镜(日本)观察试件不同破坏程度时的局部破 坏形貌。

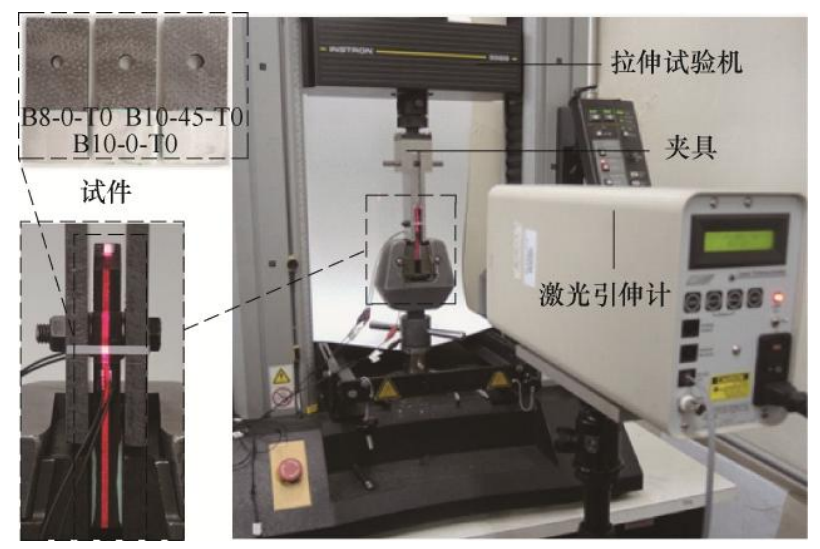

图 $2 \mathrm{C} / \mathrm{C}$ 编织复合材料螺栓连接结构挤压试验设备及夹具

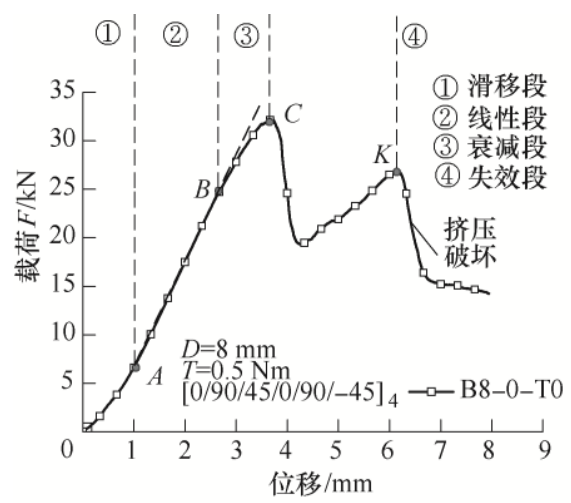

(a) 试件 B8-0-T0

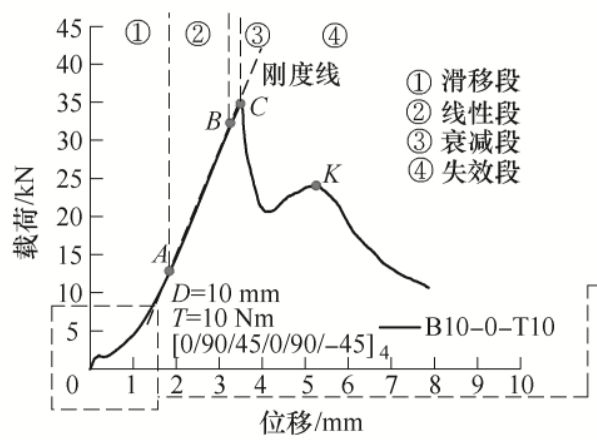

\section{2 结果与讨论}

\section{1 载荷-位移曲线}

分析 $\mathrm{C} / \mathrm{C}$ 编织复合材料典型连接结构的载荷位移曲线, 如图 3 所示。载荷-位移曲线上有三个关 键节点: $A$ 点是线性段的起点; $B$ 点为连接结构的 刚度衰减点; $C$ 点为载荷极值点。此三个关键节点 将载荷-位移曲线划分为若干个区域: (1) 滑移段, $O A$ 段为从坐标原点至 $A$ 点阶段连接结构没有紧密 配合, 试件依靠夹具与试件间的摩擦力传递载荷, 螺栓没有与试件紧密配合, 因此载荷位移曲线表现 出明显的非线性; (2) 线性段, $A B$ 阶段内连接件与 紧固件紧密配合, 由紧固件传递载荷, 载荷-位移曲 线表现为明显的线性关系，在此阶段虽然可以听到 试件在加载过程中少量的清脆断裂声，但从载荷位 移曲线中未观察到明显的刚度衰减; (3) 衰减段, $B C$ 段为衰减段, 在此阶段可以听到较多的清脆断裂 声, 且载荷位移曲线有明显的衰减, 载荷-位移曲线 在此阶段达到极值; (4) 失效段, $C$ 点之后的阶段为 结构失效阶段, 在载荷下降过程中可以听到密集的 清脆断裂声。除试件 B10-45-T0(图 3b)外，所有测 试试件均有二次峰值点 $K$ (图 3a、图 3c)。

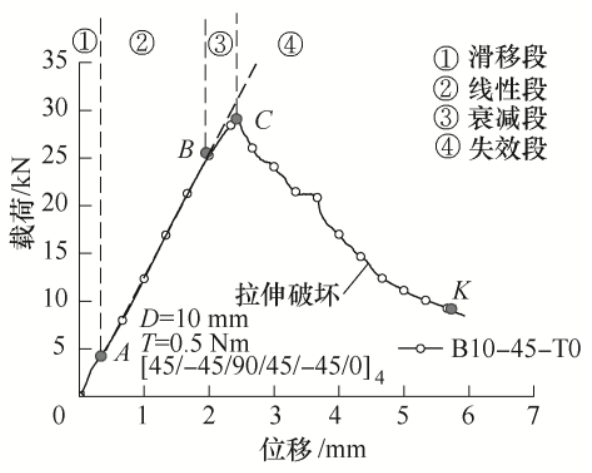

(b) 试件 B10-45-T0

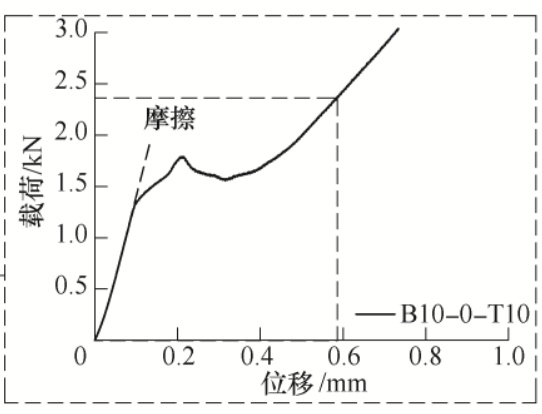

(c) 试件 B10-0-T0

图 $3 \mathrm{C} / \mathrm{C}$ 编织复合材料螺栓连接结构挤压测试典型试件的载荷-位移曲线 


\section{2 失效机理}

\subsection{1 挤压破坏}

图 4 为发生挤压破坏(B10-0-T0)和拉伸破坏 (B10-45-T0)的典型试件的破坏形貌。由图 4a 中可 以观察到试件发生挤压破坏, 主要表现为挤压区 域发生大量的纤维断裂, 并伴有大量的材料堆积。 图 5 为挤压试件 B10-0-T0 在载荷位移曲线 $C$ 点与 $K$ 点对应的破坏形貌。图中可观察到试件在两次峰 值后挤压平面均有纤维断裂、纤维屈曲与分层发 生。在一次峰值时, 损伤区域的宽度大约是垫圈 约束区域的 2 倍, 在试件达到二次峰值后损伤区

域向板端延伸，从损伤的数量到损伤的区域都有 显著的增加。

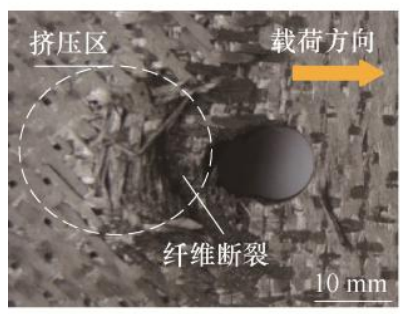

(a) 挤压破坏试件B10-0-T0

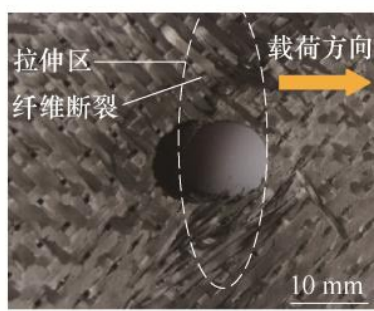

(b) 拉伸破坏试件B10-45-T0

图 $4 \mathrm{C} / \mathrm{C}$ 编织复合材料螺栓连接结构挤压测试失效模式

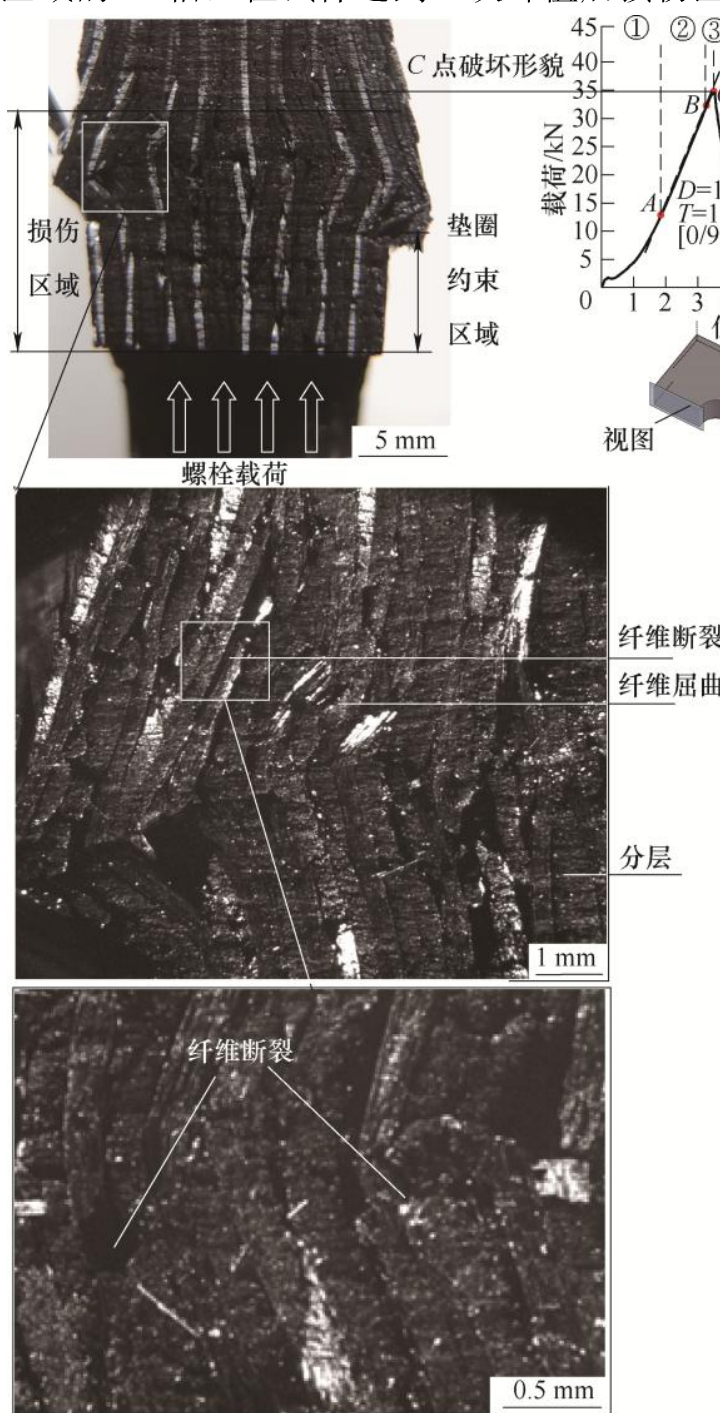

(a) $C$ 点破坏形貌

\section{图 $5 \mathrm{C} / \mathrm{C}$ 编织复合材料螺栓连接结构挤压测试典型试件 B10-0- $\mathrm{T} 0$ 破坏形貌}

试件(B10-0-T0)发生挤压破, 是连接在载荷 作用下挤压区材料渐进失效的过程。首先, 螺栓 与复合材料板的孔壁接触, 随着载荷增加孔壁最 先发生基体裂纹并不断向挤压区扩展(图 6a); 接 着, 复合材料板会有少量基体裂纹、纤维断裂 与纤维屈曲(图 6b), 由于垫片提供侧向约束垫片
下不会发生大规模的分层破坏; 最后，损伤扩展 至垫片约束范围外， $\mathrm{C} / \mathrm{C}$ 复合材料在挤压区域发 生大量的分层破坏(图 6c), 导致最终结构整体 失效。 


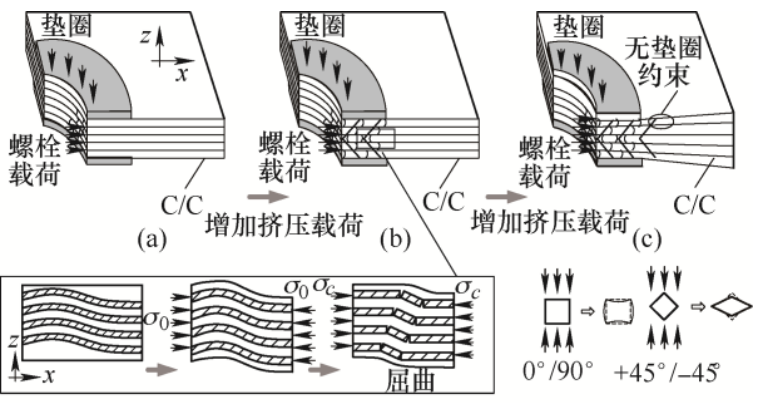

图 $6 \mathrm{C} / \mathrm{C}$ 编织复合材料螺栓连接结构挤压破坏机制图

\subsection{2 拉伸破坏}

图 4b 是试件 B10-45-T0 发生拉伸破坏时的破坏 形貌。在拉伸区域可观察到明显的纤维断裂。图 7 为挤压试件 B10-45-T0 在达到峰值载荷后的破坏形 貌。由图中可观察到视图 1 为挤压区, 可观察到少 量的纤维断裂与分层, 视图 2 为开孔截面的侧视图, 可观察到大量的分层破坏和明显的纤维断裂, 拉伸 破坏主要由拉伸面纤维断裂失效造成。

试件(B10-45-T0)发生拉伸破坏是拉伸区纤维 断裂失效所致。一方面 $45^{\circ}$ 方向的纤维铺层替换了 $0^{\circ}$ 方向纤维, 导致降低拉伸破坏平面的有效受载的纤 维含量, 另一方面 $45^{\circ}$ 纤维的增加, 加剧了在挤压 载荷下不同纤维层的变形不协调, 加速了 $\mathrm{C} / \mathrm{C}$ 复合 材料的分层(图 6d), 因此 0 度纤维体积含量的降低 结构的失效模式由挤压破坏变为拉伸破坏。

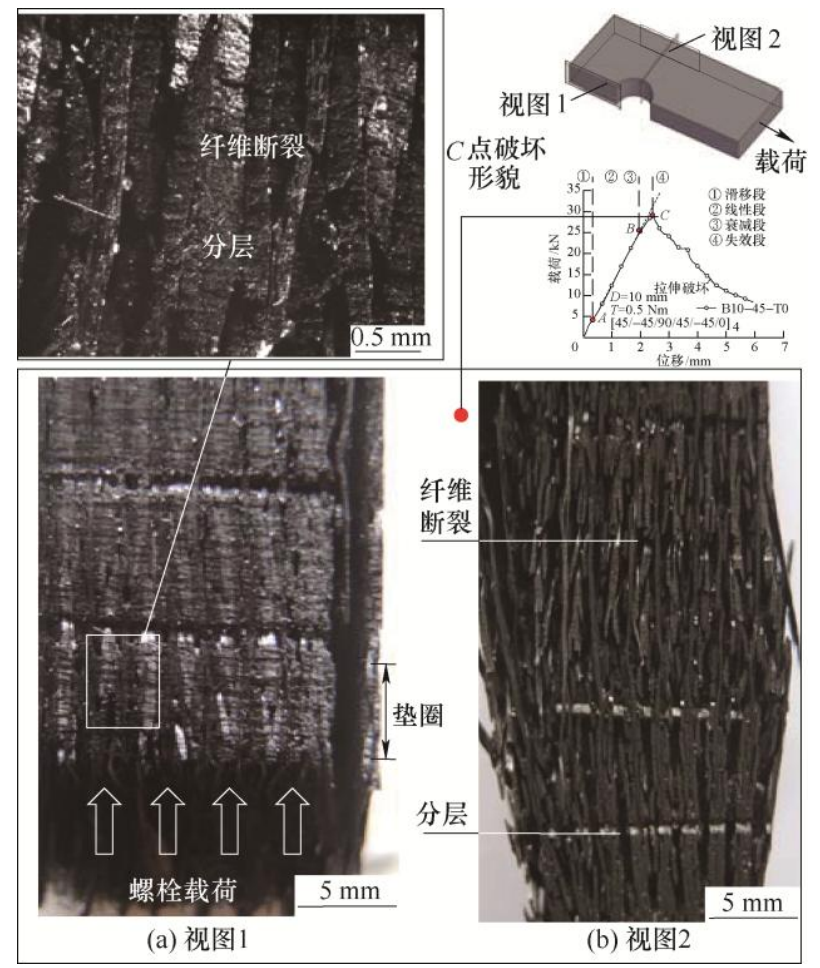

图 $7 \mathrm{C} / \mathrm{C}$ 编织复合材料螺栓连接结构挤压测试典型试件 B10-45-T0 破坏形貌

\section{3 参数讨论}

\section{1 数值模型}

采用 ABAQUS 显式分析(Explicit)建立有限元 模型, 考虑了连接模型中复杂的接触及复合材料损 伤的非线性特性。夹具、螺栓、垫片及复合材料均 采用三维缩减积分单元 C3D 8R。为了更好地模拟开 孔附近的应力分布情况, 试件开孔附近网格需加密, 加密区网格大小为 $1 \mathrm{~mm}$, 其他区域网格大小为 $2 \mathrm{~mm}$, 试件共有 99640 个网格, 试件与夹具的几何 及网格划分情况如图 8 所示。螺栓与夹具均为钢材, 在加载过程中没有发生破坏, 将其定义为线弹性材 料: 弹性模量 $E=210 \mathrm{GPa}$, 泊松比 $v=0.3$, 密度 $\rho=7.8 \times 10^{-9} \mathrm{t} / \mathrm{mm}^{3}$ 。 $\mathrm{C} / \mathrm{C}$ 编织复合材料的力学性能见 表 2 (由试件制备单位提供)。

表 $2 \mathrm{C} / \mathrm{C}$ 编织复合材料的力学性能

\begin{tabular}{lc}
\hline \multicolumn{1}{c}{ 参数 } & 数值 \\
\hline $1 、 2$ 方向弹性模量 $E_{11}, E_{12} / \mathrm{GPa}$ & 83.38 \\
3 方向弹性模量 $E_{33} / \mathrm{GPa}$ & 91.63 \\
12 面内剪切模量 $G_{12} / \mathrm{GPa}$ & 6.886 \\
$13 、 23$ 面内剪切模量 $G_{13}, G_{23} / \mathrm{GPa}$ & 6.49 \\
12 面内泊松比 $v_{12}$ & 0.0385 \\
$13 、 23$ 面内泊松比 $v_{13}, v_{23}$ & 0.0352 \\
密度 $\rho /\left(\mathrm{g} / \mathrm{cm}^{3}\right)$ & 1.815 \\
$1 、 2$ 方向拉伸强度 $S_{11}^{T}, S_{22}^{T} / \mathrm{MPa}$ & 224.4 \\
$1 、 2$ 方向压缩强度 $S_{11}^{C}, S_{22}^{C} / \mathrm{MPa}$ & 215.6 \\
3 方向拉伸强度 $S_{33}^{T} / \mathrm{MPa}$ & 314.6 \\
3 方向压缩强度 $S_{33}^{C} / \mathrm{MPa}$ & 331.1 \\
12 面内剪切强度 $S_{12} / \mathrm{MPa}$ & 33.22 \\
$13 、 23$ 面内剪切强度 $S_{13}, S_{23} / \mathrm{MPa}$ & 6.039 \\
\hline
\end{tabular}

预紧力的施加在数值模拟中通过升高垫片温度 来实现的, 垫片的线膨胀系数为 $1.35 \times 10^{-5} /{ }^{\circ} \mathrm{C}$ 。螺栓 的拧紧力矩通常分为两部分, 一部分用来克服内外 螺纹间的相互摩擦; 另一部分用来产生扭转切应力, 工程上常采用简单的公式进行估算，在模拟计算过 程中, 通过以下公式 ${ }^{[16]}$ 对计算模型施加预紧力

$$
T=k \cdot d \cdot P_{0}
$$

式中, $T$ 为扭转力矩, $k$ 为拧紧力矩系数, 对于金 属螺栓, 一般条件下取 0.2 , 润滑良好时取 $0.11 \sim 0.12$, 本文 $k$ 取值为 $0.2 。 d$ 为螺栓直径, $P_{0}$ 为螺栓预紧力。 计算出螺栓横截面拉伸正应力和扭转切应力

$$
\begin{gathered}
\sigma_{\max }^{\text {blot }}=\frac{P_{0}}{\pi d^{2} / 4}=\frac{4 T}{k d \times \pi d^{2}}=\frac{20 T}{\pi d^{3}} \\
\tau_{\max }^{b l o t}=\frac{0.5 T}{\pi d^{3} / 16}=\frac{8 T}{\pi d^{3}}
\end{gathered}
$$

将螺栓的扭转力矩 $T$ 代入式(1)可计算出螺栓预 紧力 $P_{0}$ (表 3), 将 $T$ 代入式(2), 可计算出螺栓在已知 
扭转力矩下横截面拉伸应力, 进而计算出螺栓的伸长 量, 将螺栓伸长量通过升高垫片温度实现(表 3)。

\begin{tabular}{ccccc} 
表 $\mathbf{3}$ & $\mathbf{C} / \mathbf{C}$ 编织复合材料螺栓连接挤压试件数值参数 \\
\hline $\begin{array}{c}\text { 螺栓直径 } \\
\text { 扭转力矩 }\end{array}$ & $\begin{array}{c}\text { 预紧力 } \\
d / \mathrm{mm}\end{array}$ & $\begin{array}{c}\text { 螺栓的拉伸正 } \\
\text { 垫片升高的 } \\
\text { 温度 } /{ }^{\circ} \mathrm{C}\end{array}$ \\
\hline 8 & 0.5 & 312.5 & 6.22 & 1.14 \\
10 & 0.5 & 250 & 3.18 & 0.58 \\
10 & 10 & 5000 & 63.69 & 11.67 \\
10 & 20 & 10000 & 127.39 & 23.33 \\
\hline
\end{tabular}

$\mathrm{C} / \mathrm{C}$ 编织复合材料连接结构在厚度方向上, 根 据碳纤维的铺层数量平均分配各层, 并将试件延厚 度方向剖分, 逐层定义材料方向(图 8)。试件在加载 过程中可观察到 $\mathrm{C} / \mathrm{C}$ 编织复合材料的纤维与基体不 同程度的损伤, 本文采用 VUMAT 子程序定义材料 本构模型、破坏准则和刚度衰减规则, 模拟连接结 构中纤维和基体的渐进损伤过程。子程序主要基本 的求解步骤是: 给定外载荷增量, 计算应力增量, 代入本构模型, 应用破坏准则判断是否发生破坏, 如未达到破坏, 则继续加载; 如达到破坏, 则按照 规则对材料的刚度进行衰减, 再重新以上的计算步 骤, 直至材料全部失效。

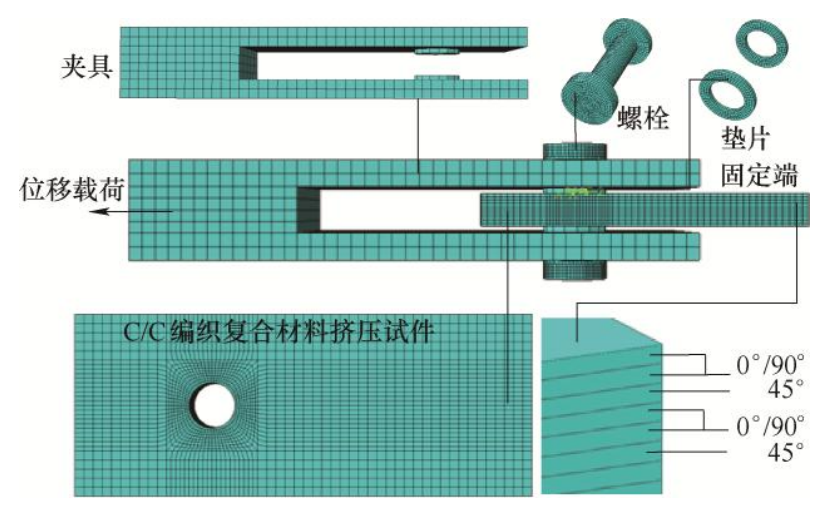

图 $8 \mathrm{C} / \mathrm{C}$ 编织复合材料双剪连接结构有限元模型

采用基于应变的 Hashin 破坏准则, 考虑了纤维 的拉伸、压缩和基体的拉伸、压缩失效模式。这主
要考虑了在连接结构中 $\mathrm{C} / \mathrm{C}$ 编织复合材料由于接触 应力变化使材料应力分布不均匀, 应力形式的失效 因子不能很好地模拟材料的渐进损伤过程。子程序 中基于应变的 $\mathrm{C} / \mathrm{C}$ 编织复合材料的失效因子 $R_{i}(i=f t, f c, m t, m c)$, 具体表示如下。

纤维拉伸失效因子

$$
R_{f t}^{2}=\left(\frac{\varepsilon_{11}}{X_{t}^{\varepsilon}}\right)^{2} \quad \varepsilon_{11}>0
$$

纤维压缩失效因子

$$
R_{f c}^{2}=\left(\frac{\varepsilon_{11}}{X_{c}^{\varepsilon}}\right)^{2} \quad \varepsilon_{11}<0
$$

基体拉伸失效因子

$$
\begin{gathered}
R_{m t}^{2}=\left(\frac{\varepsilon_{11}+\varepsilon_{33}}{Y_{t}^{\varepsilon}}\right)^{2}+\left(\frac{1}{\left.S_{23}^{\varepsilon}\right)}\right)\left(\varepsilon_{23}{ }^{2}-\frac{E_{22} E_{33}}{G_{23}{ }^{2}} \varepsilon_{22} \varepsilon_{33}\right)+ \\
\left(\frac{\varepsilon_{12}}{S_{12}^{\varepsilon}}\right)^{2}+\left(\frac{\varepsilon_{13}}{S_{13}^{\varepsilon}}\right)^{2} \quad \varepsilon_{22}+\varepsilon_{33} \geqslant 0
\end{gathered}
$$

基体压缩失效因子

$$
\begin{gathered}
R_{m c}^{2}=\left(\frac{E_{22} \varepsilon_{22}+E_{33} \varepsilon_{33}}{2 G_{12} S_{12}^{\varepsilon}}\right)^{2}+\left(\frac{\varepsilon_{22}+\varepsilon_{33}}{Y_{c}^{\varepsilon 2}}\right)\left[\left(\frac{E_{22} Y_{c}^{\varepsilon}}{2 G_{12} S_{12}^{\varepsilon}}\right)^{2}-1\right]+ \\
\frac{1}{S_{23}^{\varepsilon 2}}\left(\varepsilon_{23}^{2}-\frac{E_{22} E_{33}}{G_{23}^{2}} \varepsilon_{22} \varepsilon_{33}\right)+\left(\frac{\varepsilon_{12}}{S_{12}^{\varepsilon}}\right)^{2}+\left(\frac{\varepsilon_{13}}{S_{13}^{\varepsilon}}\right)^{2} \quad \varepsilon_{22}+\varepsilon_{33}<0(7)
\end{gathered}
$$

式中, $X_{t}^{\varepsilon} 、 X_{c}^{\varepsilon}$ 为单向板纵向拉伸强度和压缩强度 对应的应变; $Y_{t}^{\varepsilon} 、 Y_{c}^{\varepsilon}$ 为单向板横向拉伸强度和压 缩强度对应的应变; $S_{12}^{\varepsilon} 、 S_{13}^{\varepsilon} 、 S_{23}^{\varepsilon}$ 为单向板不同方向 剪切强度对应的应变; $Z_{t}^{\varepsilon}$ 为单向板外拉伸强度对应的应 变。 $X_{i}^{\varepsilon}=S_{11}^{i} / E_{11}(i=T, C), Y_{i}^{\varepsilon}=S_{22}^{i} / E_{22}(i=T, C)$, $Z_{T}^{\varepsilon}=S_{33}^{T} / E_{33}, \quad S_{12}^{\varepsilon}=S_{12} / G_{12}, \quad S_{13}^{\varepsilon}=S_{13} / G_{13}, \quad S_{23}^{\varepsilon}=$ $S_{23} / G_{23}$ 。

$\mathrm{C} / \mathrm{C}$ 编织复合材料同样采用基于应变的连续刚度 衰减方法, 退化材料性能。当材料满足 Hashin 失效准 则时, 材料发生损伤, 材料性能按照式(8)规则退化

$$
\left(\begin{array}{l}
\varepsilon_{11} \\
\varepsilon_{22} \\
\varepsilon_{33} \\
\gamma_{12} \\
\gamma_{23} \\
\gamma_{13}
\end{array}\right)=\left(\begin{array}{cccccc}
\frac{1}{E_{11}\left(1-\omega_{1}\right)} & -\frac{v_{12}}{E_{22}} & -\frac{v_{13}}{E} w_{33} & 0 & 0 & 0 \\
-\frac{v_{12}}{E_{22}} & \frac{1}{E_{2(2}(-\omega)} & -\frac{v_{23}}{E_{22}} & 0 & 0 & 0 \\
-\frac{v_{13}}{E_{33}} & -\frac{v_{23}}{E_{22}} & \left.\frac{1}{E\left({ }_{3} \frac{1}{3} \omega\right.}\right)_{3} & 0 & 0 & 0 \\
0 & 0 & 0 & \frac{1}{G_{12}\left(1-\omega_{4}\right)} & 0 & 0 \\
0 & 0 & 0 & 0 & \frac{1}{G_{23}\left(1-\omega_{5}\right)} & 0 \\
0 & 0 & 0 & 0 & 0 & \frac{1}{G_{13}\left(1-\omega_{6}\right.}
\end{array}\right)\left(\begin{array}{l}
\sigma_{11} \\
\sigma_{22} \\
\sigma_{33} \\
\tau_{12} \\
\tau_{23} \\
\tau_{13}
\end{array}\right)
$$


式中，损伤参数 $\omega_{i}(i=1,2, \cdots, 6)$ 定义为 $\omega_{1}=$ $\max \left(0.0, d_{f}\right), \omega_{2}=\max \left(0.0, d_{f}\right), \omega_{3}=\max \left(0.0, d_{m}\right)$, $\omega_{4}=\max \left(0.0, d_{f}, d_{m}\right), \omega_{5}=\max \left(0.0, d_{f}\right), \omega_{6}=\max \left(0.0, d_{f}\right)$, $d_{f}=\max \left(0.0, d_{f t}, d_{f c}\right), d_{m}=\max \left(0.0, d_{m t}, d_{m c}\right) 。$

定义损伤变量 $d_{i}\left(d_{i} \in[0,1]\right)$ 如下

$$
d_{i}=\left\{\begin{array}{ll}
0 & R_{i}<1 \\
1-\frac{1}{R_{i}} & R_{i} \geqslant 1
\end{array} i=f t, f c, m t, m c\right.
$$

损伤变量 $d_{i}(i=f t, f c, m t, m c)$ 为量纲一量表征 纤维、基体损伤程度, 当损伤变量 $d_{i}=0$ 时, 材料 完好; 当损伤变量 $d_{i}=1$ 时, 材料完全失效。代表材
料的损伤程度, 如图 9、10 所示。

\section{2 模型有效性}

通过两个标准验证数值模型的可靠性: (1) 破 坏形貌; (2) 载荷位移曲线。首先, 将数值模拟与 试验的损伤情况进行对比, 图 9 为试件 B10-0-T0 发生的破坏形貌, 数值模型很好地模拟了试件的 挤压破坏，挤压区域纤维与基体在压应力下发生 失效, 与试验结果吻合较好。图 10 为试件 B10-45-T0 的破坏形貌, 数值结果显示, 试件发生拉伸破坏, 拉伸区纤维与基体在拉应力下失效，与试验结果 吻合较好。

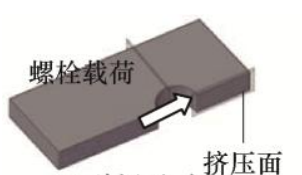

(a) 破坏平面

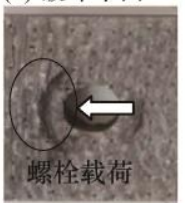

(b) 试验结果
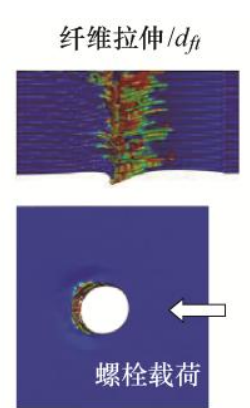

螺栓载荷
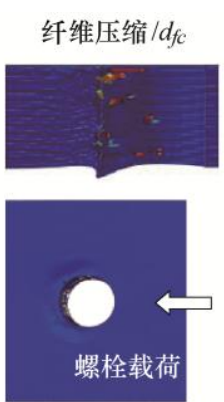

基体拉伸 $/ d_{m t}$
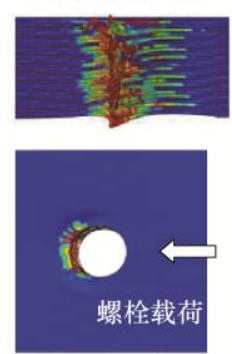

(c) 数值结果

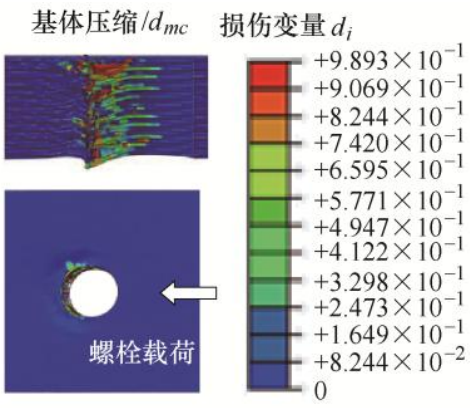

图 $9 \mathrm{C} / \mathrm{C}$ 编织复合材料螺栓连接结构试件 D10-0-T0 破坏形貌对比

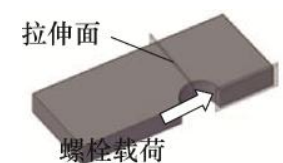

(a) 破坏平面

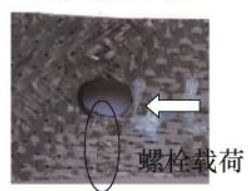

(b) 试验结果
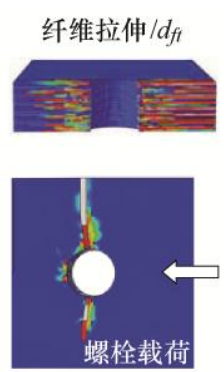

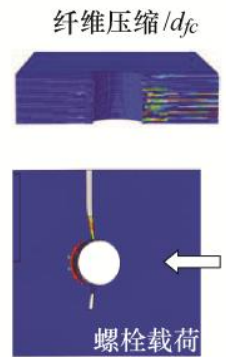

蝶栓载荷

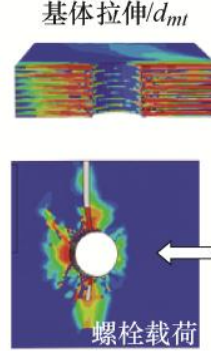

(c) 数值结果
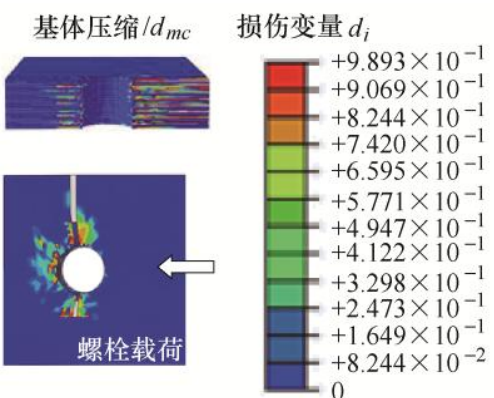

图 $10 \mathrm{C} / \mathrm{C}$ 编织复合材料螺栓连接结构试件 D10-45-T0 破坏形貌对比

图 11 典型试件载荷位移曲线的试验与数值对 比结果。结果显示, 数值模拟结果与试验曲线在达 到极限载荷前吻合较好，在达到极限载荷后二者产 生了较大的偏差。 $\mathrm{C} / \mathrm{C}$ 编织复合材料试件制备中产 生的随机初始缺陷导致结构破坏具有一定的离散性。 缺陷的数量、位置及形状等诸多不确定因素累积至 结构失效并相互耦合、放大，结构在达到峰值后的 载荷位移曲线会有一定的离散性。然而, 在数值模 型中没有考虑材料的初始缺陷, 材料按照理想模型 既定的损伤演化规律进行刚度退化。因此，在破坏 过程中随机损伤带来的影响累积到一定程度时, 数 值结果与试验结果就会产生较大的偏差。缺陷给损 伤带来的影响有待以后的研究展开进一步的讨论。
连接结构的破坏载荷数值结果误差在 $15 \%$ 范围内, 数值模型可以有效地预报结构的破坏载荷。

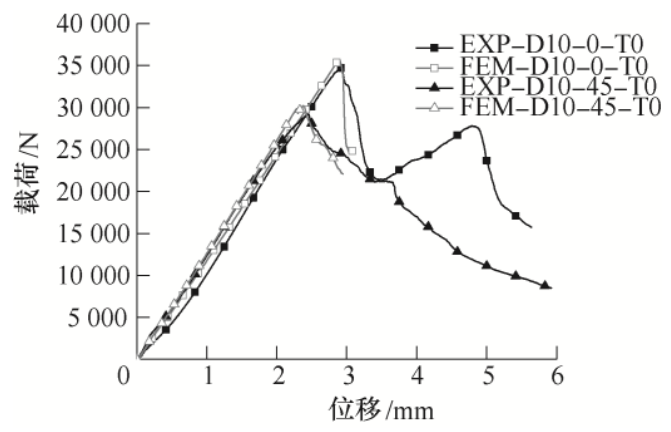

图 $11 \mathrm{C} / \mathrm{C}$ 编织复合材料螺栓连接结构载荷-位移曲线

由以上失效模式及载荷位移曲线的对比结果可 知, 数值模型可以有效地模拟 $\mathrm{C} / \mathrm{C}$ 编织复合材料螺 
栓连接的失效模式和破坏载荷。可以利用该数值模 型展开更广泛的参数研究, 为结构设计提供一定的 理论依据。

\section{3 堆叠顺序对挤压强度的影响}

考虑了以下铺层因素: (1) $0^{\circ}$ 纤维含量; (2) 对 称性。利用数值模型, 分析开孔直径 $10 \mathrm{~mm}$, 预紧 力 $0.5 \mathrm{~N} \cdot \mathrm{m}$ 时, 铺层参数对连接破坏的影响, 数值 计算结果如图 12 所示。对称铺层破坏载荷均高于循 环铺层情况, 准各向同性铺层的连接破坏载荷高于 正交铺层连接。 $[-45 / 45 / 0 /-45 / 45 / 90]_{4}$ 的破坏载荷最 低, 与试验结果吻合。这是由于 $0^{\circ}$ 方向纤维含量少 致螺栓连接中拉伸区域承载纤维少, 从而降低净截 面拉伸强度, 较易出现拉伸破坏。

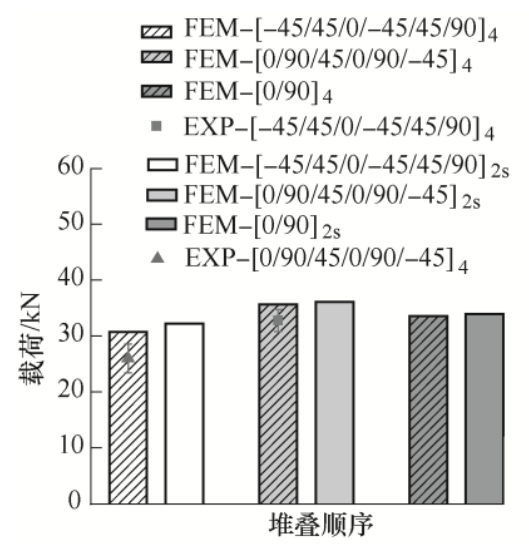

图 12 铺层顺序对 $\mathrm{C} / \mathrm{C}$ 编织复合材料双剪螺栓 连接结构破坏载荷的影响

\section{4 几何尺寸对挤压强度的影响}

分析 $\mathrm{C} / \mathrm{C}$ 编织连接结构中螺栓孔径对破坏载荷 的影响, 利用数值模型模拟分析给出了孔径对破坏 载荷影响的分析结果, 如图 13a 所示。由图中结果 可知, 当板宽、板厚和预紧力一定时, 随着开孔直 径 $D$ 的增加连接的破坏载荷先增加后降低。这是由 于开孔过大使板边距降低, 且失效模式由挤压破坏 变为拉伸破坏, 挤压应力降低。同时也表明, 在孔 径增大的初期, 破坏载荷随孔径的增大而增大, 螺 栓与螺孔的接触面积随孔径的增大而增大, 破坏载 荷随之提高, 之后由于失效模式的转变(由挤压破坏 转变为拉伸破坏, 板边距减小), 破坏载荷会降低。 在 $D \approx 14 \mathrm{~mm}$ 处失效模式由挤压破坏转变为拉伸破 坏。针对板宽 $W=50 \mathrm{~mm}$, 板厚 $t=10 \mathrm{~mm}$ 的 $\mathrm{C} / \mathrm{C}$ 编 织双剪螺栓连接结构, 开孔直径 $D \approx 14 \mathrm{~mm}$ 最优, 此时 $D / t \approx 1.4$ 。

为了分析 $\mathrm{C} / \mathrm{C}$ 编织连接结构中连接的板厚 $t$ 对 破坏载荷和挤压应力的影响, 经模拟分析给出了板 厚对破坏载荷及挤压应力影响的分析结果, 如图
$13 \mathrm{~b}$ 所示。由图中结果可观察到, 当开孔直径 $D=10 \mathrm{~mm}$, 板宽 $W=50 \mathrm{~mm}$ 时, 随着板厚的增加连 接的破坏载荷增加, 而挤压应力先增加后降低, 失 效模式始终为挤压破坏。当开孔直径为 $10 \mathrm{~mm}$ 时, 板厚 $t=6 \mathrm{~mm}$ 时, 其破坏时挤压应力达最大值, $D / t$ 为 1.7 。综上考虑板宽与板厚的关系, 建议 $D / t$ 取值 范围为 $1.2 \sim 1.8$ 。

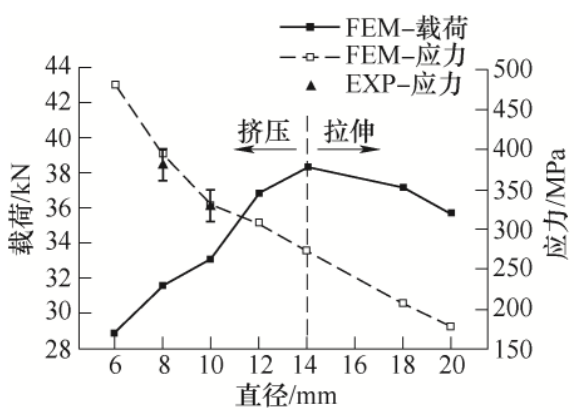

(a) 直径对破坏载荷/挤压应力的影响

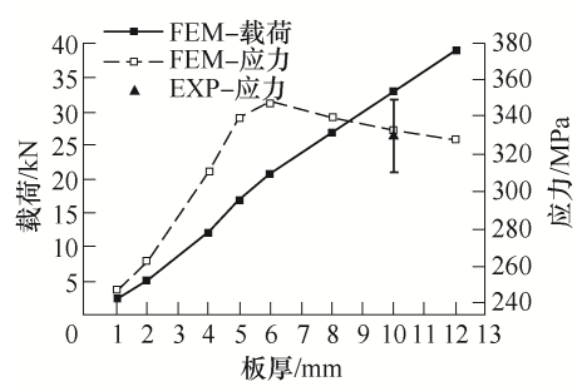

(b) 板厚对破坏载荷/挤压应力的影响

图 13 几何尺寸对 $\mathrm{C} / \mathrm{C}$ 编织复合材料双剪螺栓 连接破坏载荷和挤压应力的影响

\section{5 预紧力对挤压强度的影响}

预紧力对连接结构影响问题也是连接结构力学 特性分析当中必须要考虑的, 为了进一步分析 $\mathrm{C} / \mathrm{C}$ 编织连接结构中对螺栓施加的预紧力对破坏载荷及 挤压应力的影响, 这里结合试验结果, 经模拟分析 给出了预紧力对破坏载荷影响的分析结果, 如图 14 所示。由图 14 中可知, 随着预紧力的增大破坏载荷 和挤压应力均增大, 但涨幅在 5\%左右, 因此预紧力 对 $\mathrm{C} / \mathrm{C}$ 编织连接结构的最终破坏载荷影响不大。

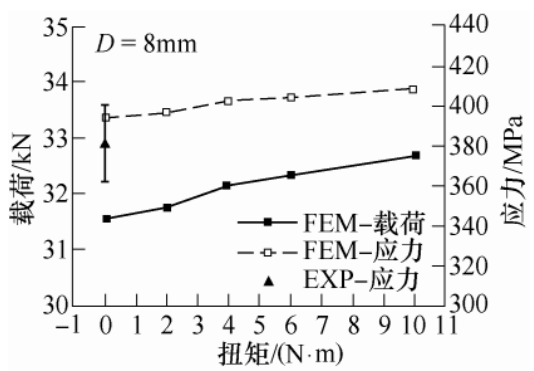

图 14 预紧力对 $\mathrm{C} / \mathrm{C}$ 编织复合材料双剪螺栓 连接破坏载荷和挤压强度的影响 


\section{6 破坏包线图}

破坏包线图是经验分析方法预报多螺栓连接强 度和失效模式的依据, 最初由 $\mathrm{SMITH}^{[17]}$ 提出。本文 以 $[0 / 90 / 45 / 0 / 90 /-45]_{4}$ 铺层为例, 借助确定的数值模 型绘制 $\mathrm{C} / \mathrm{C}$ 编织复合材料连接的破坏包线图, 为多 螺栓连接强度预报提供理论支持。一般情况下, 在 拉伸载荷下挤压应力与旁路应力呈线性关系, 载荷 率(挤压载荷/应力与旁路载荷/应力的比值)较高时 发生挤压破坏, 当载荷率较低时, 失效模式会转变 为拉伸破坏。这里通过数值模型确定 $\mathrm{C} / \mathrm{C}$ 编织复合 材料连铺层为 $[0 / 90 / 45 / 0 / 90 /-45]_{4}$ 时, 其连接在拉伸 载荷作用下的破坏包络图, 如图 15 所示。

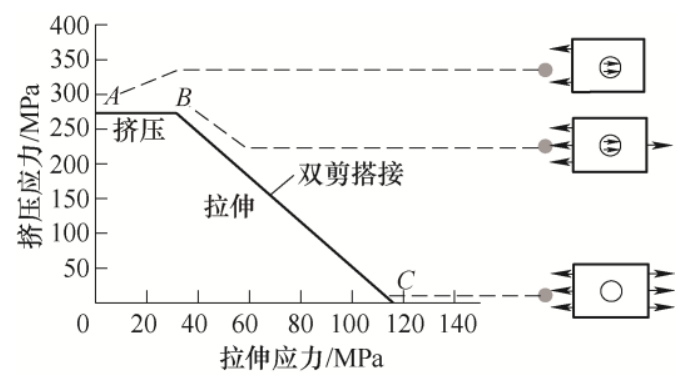

图 $15 \mathrm{C} / \mathrm{C}$ 编织复合材料双剪螺栓连接结构破坏包线图

图 15 中 $A B$ 线段为挤压强度截止线, $A$ 点应力 值可以通过式(10)确定

$$
\sigma_{A}=\sigma_{b r u}=\frac{F_{b r u}}{D t}
$$

式中, $\sigma_{b r u}$ 为挤压破坏强度, $F_{b r u}$ 为发生挤压破坏的 破坏载荷, 利用之前验证的数值模型, 结合几何参 数的讨论结果, 确定发生挤压破的几何尺寸, 通过 数值模拟确定双剪螺栓连接结构发生挤压破坏时的 破坏载荷 $F_{b r u}$ 。 $D 、 t$ 分别代表开孔直径和板厚。图 中 $C$ 点为开孔板拉伸破坏载荷与净截面之比, 可通 过模拟开孔板拉伸破坏得到该应力值。 $B C$ 线段代表 拉伸载荷作用下，挤压应力和旁路应力共同作用下 的破坏包线, 由式(11)确定

$$
K_{b c} \sigma_{b r}+K_{t c} \sigma_{n e t}=\sigma_{b}
$$

式中, $\sigma_{b}$ 为无开孔时材料的拉伸(压缩)强度, 见表 $2, \sigma_{b r}$ 为研究对象受载孔挤压应力, 即破坏包线图 的纵坐标轴, $\sigma_{\text {net }}$ 为由旁路载荷引起的净截面拉伸 应力, 即破坏包线图的横坐标轴, $K_{b c}$ 为受载孔挤 压应力集中减缩系数, $K_{t c}$ 为开孔拉伸应力集中减缩 系数, 其定义式为

$$
\left\{\begin{array}{l}
K_{b c}=\frac{1+C_{r e}\left[K_{b e}(W / D-1)-1\right]}{W / D-1} \\
K_{t c}=1+C_{r e}\left(K_{t e}-1\right)
\end{array}\right.
$$

式中, $C_{r e}$ 为应力集中减缓因子, 一般取值为 $0 \sim 1$
之间，与材料性能相关，且假设开孔试件与连接试 件具有相同的应力集中减缓因子。 $K_{t e}$ 和 $K_{b e}$ 分别为 各向同性材料受载孔拉伸和挤压弹性应力集中系数, 可通过式(13)计算得到

$$
\begin{aligned}
& K_{t e}=2+\left(1-\frac{D}{W}\right)^{3} \quad K_{b e}=1+\frac{2}{\frac{W}{D}-1}-\frac{1.5 \theta}{\frac{W}{D}+1} \\
& \text { 当 } E / W \leqslant 1 \text { 时 } \\
& \qquad \theta=1.5-\frac{0.5}{E / W}
\end{aligned}
$$

当 $E / W>1$ 时

$$
\theta=1
$$

式中, $E$ 为端距, $W$ 为板宽。与材料相关的应力集 中减缓因子 $C_{r e}$ 是包线图绘制的关键, 该值通过数值 计算获得拉伸破坏强度，将其代入式(11)、式(12) 可计算出 $C_{r e}=0.4821$ 。

\section{4 结论}

（1）挤压破坏是一个渐进损伤的过程，基体裂 纹由孔壁逐步向板端扩展，在挤压区域发生纤维由 压应力造成屈曲而后断裂, 垫圈为结构提供了一定 的侧向约束, 可以明显地抑制挤压区的分层失效。

(2) $45^{\circ}$ 纤维在压应力下与 $0^{\circ}$ 纤维变形不协调, 会导致结构分层严重; 当载荷方向纤维体积含量降低 时, 连接的失效模式由挤压破坏变为拉伸破坏。

(3) 利用确定的数值模型对 $\mathrm{C} / \mathrm{C}$ 编织复合材料 双剪搭接螺栓连接的铺层顺序、几何参数、预紧力 对连接性能的影响进行了分析。分析结果显示连接 结构采用对称铺层方式较循环铺层方式的连接性能 好, 准各向同性铺层形式较正交铺层形式的连接性 能好; $D / t$ 建议取值范围为 $1.2 \sim 1.8$; 预紧力对连 接破坏载荷(强度)提高不超过 $5 \%$; 并给出 $\mathrm{C} / \mathrm{C}$ 编织 复合材料双剪螺栓连接结构的破坏包线图。

\section{参 考 文 献}

[1] PENG Lina, HE Guoqiang, LI Jiang, et al. Effect of combustion gas mass flow rate on carbon/carbon composite nozzle ablation in a solid rocket motor[J]. Carbon, 2012, 50: 1554-1562.

[2] TANG Yuling, ZHOU Zhengong, PAN Shidong, et al. Mechanical property and failure mechanism of $3 \mathrm{D}$ carbon-carbon braided composites bolted joints under unidirectional tensile loading[J]. Materials \& Design, 2015, 65(65): 243-253. 
[3] LIU Jialin, LIU Jiayi, JIE Mei, et al. Investigation on manufacturing and mechanical behavior of all-composite sandwich structure with Y-shaped cores[J]. Composites Science and Technology, 2018，159: 87-102.

[4] 王波, 矫桂琼. 三维编织 $\mathrm{C} / \mathrm{SiC}$ 复合材料剪切和弯曲性 能的实验研究[J]. 机械强度，2007，29(1)：44-47.

WANG Bo, JIAO Guiqiong. Shear and bending experimental investigation of three dimensionally braided C/SiC composites[J]. Journal of Mechanical Strength, 2007, 29(1): 44-47.

[5] 郭洪宝, 王波, 甄文强, 等. $2 \mathrm{D}-\mathrm{C} / \mathrm{SiC}$ 复合材料开孔件 拉伸强度有限元计算 $[\mathrm{J}]$. 复合材料学报, 2014, 31(2): 448-455.

GUO Hongbao, WANG Bo, ZHEN Wenqiang, et al. FEM calculation about tensile strength of 2D-C/SiC composites with circular holes[J]. Acta Materiae Compositae Sinica, 2014, 31(2): 448-455.

[6] 李俊, 矫桂琼, 王波, 等. 二维编织 $\mathrm{C} / \mathrm{SiC}$ 复合材料非 线性损伤本构模型与应用[J]. 复合材料学报, 2013, 30(1): 165-171.

LI Jun, JIAO Guiqiong, WANG Bo, et al. A non-linear damage constitutive model for $2 \mathrm{D}$ woven $\mathrm{C} / \mathrm{SiC}$ composite material and its application[J]. Acta Materiae Compositae Sinica, 2013, 30(1): 165-171.

[7] 唐玉玲, 泮世东, 周振功, 等. 含孔和切口 $\mathrm{C} / \mathrm{C}$ 编织复 合材料静态拉伸下的力学性能[J]. 复合材料学报, 2013 , 30(3): 87-92.

TANG Yuling, PAN Shidong, ZHOU Zhengong, et al. Mechanical properties analysis of 3D $\mathrm{C} / \mathrm{C}$ braided composite opening structures under static tension[J]. Acta Materiae Compositae Sinica, 2013， 30(3): 87-92.

[8] ZHAI Yunong, LI Xiaoqiang, WANG Liang, et al. Three-dimensional layer-by-layer stress analysis of single-lap, countersunk composite joints with varying joining interface conditions $[\mathrm{J}]$. Composite Structures, 2018, 202: 1021-1031.

[9] COOPER C, TURVEY G J. Effects of joint geometry and bolt torque on the structural performance of single bolt tension joints in pultruded GRP sheet material[J]. Composite Structures, 1995，32(1): 217-226.

[10] AKTAS A, DIRIKOLU M H. The effect of stacking sequence of carbon epoxy composite laminates on pinned-joint strength[J]. Composite Structures, 2003, 62(1): 107-111.

[11] EGAN B, MCCARTHY C T, MCCARTHY M A, et al Stress analysis of single-bolt, single-lap, countersunk composite joints with variable bolt-hole clearance[J]. Composite Structures, 2012, 94(3): 1038-1051.

[12] ZHAI Yunong, LI Dongsheng, LI Xiaoqiang, et al. An experimental study on the effect of bolt-hole clearance and bolt torque on single-lap, countersunk composite joints[J]. Composite Structures, 2015， 127: 411-419.

[13] PEARCE G M K, JOHNSON A F, HELLIER A K, et al A study of dynamic pull-through failure of composite bolted joints using the stacked-shell finite element approach[J]. Composite Structures, 2014, 118(1): 86-93.

[14] 何龙, 李成, 朱红红. 连接结构的孔边强化层对复合材 料含孔板失效过程的影响[J]. 机械工程学报, 2011, 47(12): 43-47.

HE Long, LI Cheng, ZHU Honghong. Influence of hole strengthening layer on the failure process of composite material plate with a hole in connection structures[J]. Journal of Mechanical Engineering, 2011, 47(12): 43-47.

[15] Composite Materials ASTM International. ASTM D5961/D5961M-05e1 Standard test method for bearing response of polymer-matrix composite laminates[S]. West Conshohocken: PA, 2005

[16] TANG Yuling, ZHOU Zhengong, PAN Shidong, et al. Mechanical and failure behavior of three-dimensional six-directional braided composites bolted joint[J]. Journal of Reinforced Plastics \& Composites, 2017, 36(10): 739-753.

[17] SMITH H L J. Bolted joints in graphite/epoxy composites[R]. Southern California: Technical Report NASA CR-144899, National Aeronautics and Space Administration, 1977.

作者简介: 唐玉玲, 女, 1981 年出生, 博士。主要研究方向为复合材料 及其结构的力学性能分析。

E-mail: tangyuling@tust.edu.cn 周振功(通信作者), 男, 1963 年出生, 博士, 教授, 博士研究生导师。 主要研究方向为复合材料及其结构的力学性能分析、热防护结构的设计 与分析。

E-mail: zhouzhg@hit.edu.cn 